\title{
SOME EXAMPLES OF CALABI-YAU MANIFOLDS
}

\author{
NDRIÇIM SADIKAJ AND ANILA DUKA
}

\begin{abstract}
The space-time in String Theory is often described by means of a mathematical object called manifold. Manifolds are very important objects from the mathematical and the physics point of view, not only in String Theory. Calabi-Yau manifolds are complex manifolds, and they exist in any even dimension.

The simplest examples of Calabi-Yau manifolds have one complex dimension. Some simple examples of non compact Calabi-Yau two-folds, which have two complex dimensions are $\mathbb{C}^{2}=\mathbb{C} \times \mathbb{C}, \mathbb{C} \times T^{2}$.

$K 3$ and $T^{4}$ are two examples of four-dimensional compact Kähler manifolds for which they exist.

Examples of a Calabi-Yau n-folds can be constructed as a submanifold of $\mathbb{C} P^{n+1}$ for all $n>1$.
\end{abstract}

\section{INTRODUCTION}

Calabi-Yau manifolds are compact, complex Kähler manifolds that have trivial first Chern classes (over R). In most cases, we assume that they have finite fundamental groups. By the conjecture of Calabi (1957) proved by Yau $(1977 ; 1979)$, there exists on every Calabi-Yau manifold a Kähler metric with vanishing Ricci curvature. Currently, research on Calabi-Yau manifolds is a central focus in both mathematics and mathematical physics. It is partially propelled by the prominent role the Calabi-Yau threefolds play in superstring theories.

\section{Definition of Calabi-Yau manifolds}

2.1. The Ricci tensor of Calabi-Yau manifolds. Given any Kähler metric, one defines its full curvature tensor by certain expressions of covariant derivatives of the metric; the Ricci curvature is a partial contraction of the full curvature tensor. This gives a tensor which is of the same type as the Kähler form. In local coordinates,

$$
R i c=\sqrt{-1} \sum R_{i \bar{j}} d z^{i} \wedge d \bar{z}^{j}
$$

2000 Mathematics Subject Classification. 05C10, 45654.

Key words and phrases. string teory, manifold, Calabi-Yau manifolds, Hodge numbers, Chern class, Hodge diamond. 
2.2. The Calabi conjecture. According to a well-known theorem of Chern, the Ricci form divided by $2 \pi$ is a $(1,1)$-form that represents the first Chern class of a compact complex manifold. Rooted in his attempt to find canonical Khler metrics for a Kähler manifold, in 1954, E. Calabi (Calabi, 1957) proposed his celebrated conjecture.

Conjecture: To every closed (1,1)-form $\frac{1}{2 \pi} C_{1}(X)$ representing the first Chern class $c_{1}(X)$ of a Kähler manifold $\mathrm{X}$, there is a unique Kähler metric in the same Kähler class whose Ricci tensor (form) is the closed (1,1)-form $C_{1}(X)$.

In case the complex manifold has vanishing first Chern class, the zero form represents the first Chern class of the manifold. The Calabi conjecture implies the existence of a unique Ricci-flat Kähler metric in every Kähler class.

2.3. Yau's theorem. In 1976, Yau (Yau, 1977; Yau, 1979) proved the Calabi conjecture by solving the complex Monge-Ampère equation for a real valued function $\phi$

$$
\operatorname{det}\left(g_{i \bar{j}}+\frac{\partial^{2} \phi^{j}}{\partial z^{i} \partial \bar{z}}\right)=e^{f} \operatorname{detg}_{i \bar{j}}
$$

where $e^{f}$ is any smooth function of average 1 and $g_{i j} \partial_{i} \partial_{\bar{j}} \phi$ is required to be positive definite. The solution $\phi$ of the above equation ensures that the new Kähler metric

$$
\omega+\sqrt{-1} \partial \bar{\partial} \phi
$$

can attain any Ricci (curvature) form in the class referred to in the Calabi conjecture.

2.4. Calabi-Yau manifolds and Calabi-Yau metrics. The first application to Yau's proof of Calabi conjecture is the existence of Ricci-flat Kähler metric on every compact complex Kähler manifold with trivial canonical class. (Trivial canonical class is equivalent to the existence of a nowhere vanishing holomorphic volume form, which is equivalent to that the top wedge power of the holomorphic cotangent bundle is the trivial line bundle.) The converse is also true: any Ricci-flat simply connected Kähler manifold has trivial canonical line class. This proves the existence and provides a criterion for Kähler Calabi-Yau manifolds.

By convention, Calabi-Yau manifolds exclude those with infinite fundamental groups. The Ricci-flat metrics on Calabi-Yau manifolds are called Calabi-Yau metrics. 


\section{Examples of Calabi-Yau manifolds}

3.1. Calabi-Yau one-folds. The simplest examples of Calabi-Yau manifolds have one complex dimension.

3.1.1. Complex plane. A simple noncompact example is the complex plane $\mathbb{C}$ described in terms of the coordinates $(z, \bar{z})$. It can be described in terms of a flat metric

$$
d s^{2}=|d z|^{2}
$$

and the holomorphic one-form is

$$
\Omega=d z
$$

3.1.2. Two-torus $T^{2}$. The only compact CalabiYau one-fold is the twotorus $T^{2}$, which can be described with a flat metric and can be thought of as a parallelogram with opposite sides identified.The Ricci-flat metric on a torus is actually a flat metric, so that the holonomy is the trivial group $S U(1)$. A one-dimensional Calabi-Yau manifold is a complex elliptic curve, and in particular, algebraic.

\subsection{Calabi-Yau two-folds.}

3.2.1. Noncompact examples. Some simple examples of noncompact CalabiYau two-folds, which have two complex dimensions, can be obtained as products of the previous two manifolds: $\mathbb{C}^{2}=\mathbb{C} \times \mathbb{C}, \mathbb{C} \times T^{2}$.

3.2.2. Compact examples: $T^{4}, K 3 . K 3$ and $T^{4}$ are the only two examples of four-dimensional compact Kähler manifolds for which they exist. As a result, these manifolds are the only examples of CalabiYau two-folds.

3.2.3. Orbifold limit of $K 3$. A singular limit of $K 3$, which is often used in string theory, is an orbifold of the $T^{4}$. This has the advantage that it can be made completely explicit. Consider the square $T^{4}$ constructed by taking $\mathbb{C}^{2}$ and imposing the following four discrete identifications:

$$
z^{a} \sim z^{a}+1 \quad z^{a} \sim z^{a}+i, \quad a=1,2 .
$$

There is a 2 isometry group generated by

$$
I:\left(z^{1}, z^{2}\right) \rightarrow\left(-z^{1},-z^{2}\right) .
$$

This $\mathbb{Z}_{2}$ action has 16 fixed points, for which each of the $z^{a}$ takes one of the following four values

$$
0, \quad \frac{1}{2}, \quad \frac{i}{2}, \quad \frac{i+1}{2}
$$


Therefore, the orbifold $T^{4} / \mathbb{Z}_{2}$ has 16 singularities. These singularities can be repaired by a mathematical operation called blowing up the singularities of the orbifold.

The singular points of the orbifold described above can be "repaired" by the insertion of an Eguchi-Hanson space. The way to do this is to excise a small ball of radius a around each of the fixed points.

3.2.4. Hodge numbers of K3. Hodge numbers of the Eguchi-Hanson space are $h^{0,0}=h^{1,1}=h^{2,2}=1$ The Eguchi-Hanson spaces contribute a total of 16 generators to $H^{1 ; 1}$, one for each of the 16 spaces used to blow up the singularities. Moreover, on the $T^{4}$ the following four representatives of $H^{1 ; 1}$ cohomology classes survive the $\mathbb{Z}_{2}$ identifications:

$d z^{1} \wedge d \bar{z}^{1}, d z^{1} \wedge d \bar{z}^{2}, d z^{2} \wedge d \bar{z}^{1}, d z^{2} \wedge d \bar{z}^{2}$

This gives in total $h^{1 ; 1}=20$. In addition, there is one $H^{2 ; 0}$ class represented by $d z^{1} \wedge d z^{2}$ and one $H^{2 ; 0}$ class represented by $d \bar{z}^{1} \wedge d \bar{z}^{2}$. As a result, the Hodge numbers of $K 3$ are given by the Hodge diamond

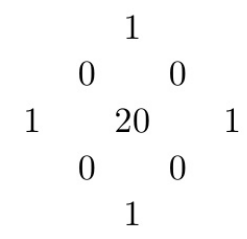

Thus, the nonzero Betti numbers of $K 3$ are $b_{0}=b_{4}=1, b_{2}=22$, and the Euler characteristic is $\chi=24$.

3.3. Calabi-Yau three-folds. In three complex dimensions, classification of the possible Calabi-Yau manifolds is an open problem, although Yau suspects that there is a finite number of families.

A Calabi-Yau 3-fold is a Kähler manifold (M,J,g) of complex dimension 3 with a covariant constant holomorphic volume form $\Omega$ such that it satisfies

$$
\omega^{3}-\frac{3 i}{4} \Omega \wedge \bar{\Omega}
$$

where $\omega$ is the Kähler form of $\mathrm{g}$. We say that $(J, \omega, \Omega)$ constitutes a Calabi-Yau structure on $\mathrm{M}$ and write a Calabi-Yau manifold as a quadruple $(M, J, \omega, \Omega)$.

Another equivalent way of defining a Calabi-Yau 3-fold is to require that the Riemannian 6-fold $(M, g)$ has holonomy group $H o l(g)$ contained in $S U(3)$. One can then show that $\mathrm{M}$ admits a holomorphic volume form satisfying the normalization formula. These are the most important CalabiYau manifolds in string theory applications.

We have already shown that the Hodge numbers of Kähler manifolds satisfy

$$
h^{p, q}=h^{3-p, 3-q}
$$


and a complex conjugation duality

$$
h^{p, q}=h^{q, p}
$$

For CalabiYau manifolds, there is a further duality, sometimes called holomorphic duality.

The Hodge diamond takes the form:

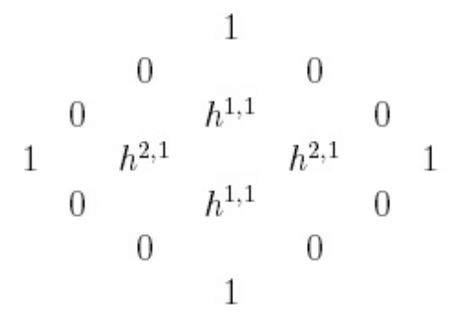

3.4. Example of Calabi-Yau threefolds. We will now study in some details two particular examples of CalabiYau threefolds: the quintic in $\mathbb{C P}^{4}$, and the Tian-Yau manifold. Both examples have been very important in the history of string theory.

Through these two examples we will study in more generality complete intersection manifolds in complex projective spaces and products thereof. But to start with we need to know the Chern classes of the complex projective spaces $\mathbb{C P}^{m}$.

Chern class of $\mathbb{C P}^{m}$

The homogeneous coordinates $z_{i}$ of $\mathbb{C}^{m+1}$ are sections of the hyperplane line bundle L. Thus, the holomorphic tangent bundle of $\mathbb{C}^{m+1}$ is spanned by tangent vectors $s_{i}(z) \frac{\partial}{\partial z_{i}}$, where the $s_{i}$ are any sections ofL. Now, on $\mathbb{C P}^{m}$, the holomorphic tangent bundle. We have to take equivalence classes with respect to overall rescaling, since overall rescaling is trivial in $\mathbb{C P}^{m}$. We find that

$$
c\left(\mathbb{C P}^{m}\right)=(1+x)^{m+1}
$$

\section{Calabi-Yau condition for complete intersection manifolds}

We now want to see what the CalabiYau condition is for complete intersection manifolds. We look at Calabi-Yau hypersurfaces in complex projective spaces. Let $\mathrm{X}$ be a smooth hypersurface in $\mathbb{C P}^{m}$ defined as the zero-locus of a degree d polynomial $\mathrm{p}$. The Chern class of $\mathrm{X}$ is

$$
c(X)=\frac{(1+x)^{m+1}}{1+d x}
$$

The first Chern class is

$$
c_{1}(X)=[(m+1)-d] x
$$


Therefore, we have found an explicit realization of the Calabi-Yau condition for hypersurfaces; the condition $c_{1}=0$ implies a condition on the degree of the polynomial equation, $d=m+1$. If we want a CalabiYau threefold, we have $m=4$, and therefore $\mathrm{X}$ must be given by the zero-locus of a degree 5 polynomial, that is a quintic in $\mathbb{C P}^{4}$. It is straightforward to generalize the above computation to complete intersection manifolds $\mathrm{Y}$ given by the zero-locus of a finite number of polynomials in $\mathbb{C P}^{m}$.

The total Chern class (using the fact that $c_{1}=0$ ), we find

$$
C(Y)=1+\frac{1}{2}\left[\left(\sum_{i=1}^{l} d_{i}^{2}\right)-(n+1)\right] x^{2}-\frac{1}{3}\left[\left(\sum_{i=1}^{l} d_{i}^{3}\right)-(n+1)\right] x^{3}
$$

This result will be useful for the computation of the Euler characteristic through the integration of the third Chern class over the manifold Y.

3.4.1. The quintic in $\mathbb{C P}^{4}$. We will now concentrate on the quintic $\mathrm{Q}$ in $\mathbb{C P}^{4}$, which is given by a polynomial equation of degree 5 in the homogeneous coordinates of $\mathbb{C P}^{4}$. According to the results of the previous section, we have that

$$
c(Q)=1+10 x^{2}-40 x^{3}
$$

To find its Euler characteristic, we must integrate

$$
-40 x^{3}
$$

over Q.

Using Poincarè duality and de Rhams theorems relating homology and cohomology and we find that

$$
\chi(Q)=\int_{Q} c_{3}(Q)=\int_{Q}\left(-40 x^{3}\right)=\int_{\mathbb{C P}^{4}}\left(-40 x^{3}\right) \wedge(50 x)=-200
$$

Now to pursue the study of the quintic further we must determine its Hodge numbers.

For the quintic in $\mathbb{C P}^{4}$, there are initially 126 parameters. The group of holomorphic automorphisms of $\mathbb{C P}^{m}$ being

$$
P G L(m+1, \mathbb{C})
$$

of them can be removed by an homogeneous linear change of variables. Moreover, one parameter corresponds to overall rescaling.

Hence, there are $h^{2,1}(Q)=126-(25-1)-1=101$ parameters describing the complex structure of Q. Since $\chi=2\left(h^{1,1}-h^{2,1}\right)$ we have that $h^{1,1}(Q)=$ 1 i.e. there is only one Ricci-flat Kähler form on the quintic. Hence the Kähler structure moduli space is one-dimensional. 
To summarize our result the quintic $\mathrm{Q}$ in $\mathbb{C P}^{4}$ has Euler class $\chi=-200$ and Hodge diamond

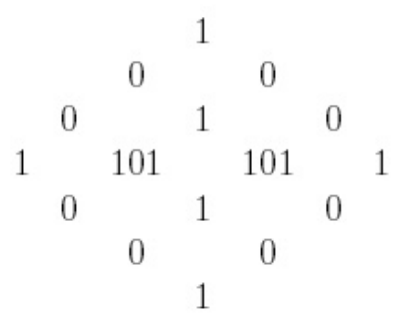

Now we know that Q is a Calabi-Yau manifold, and we studied it using Chern classes. We will now construct a holomorphic volume form $\Omega$ on $\mathrm{Q}$. Alternatively, we could have started our study of the quintic by attempting a direct construction of an holomorphic volume form, and show that way that $\mathrm{Q}$ is indeed CalabiYau. By the residue theorem we find

$$
\Omega=\frac{1}{2 \pi}\left(\frac{\sum_{\mu=1}^{4} d z_{1} \wedge d z_{2} \wedge \ldots \wedge d z_{\mu} \wedge \ldots \wedge d z_{4}}{\partial Q / \partial z_{0}}\right)
$$

which is a nowhere vanishing holomorphic $(3,0)$-form on $Q=0$. We have found an holomorphic volume form $\Omega$ on Q; therefore as we saw in the previous section all other holomorphic $(3,0)$-forms are constant multiples of $\Omega$.

3.4.2. The Tian-Yau manifold. In order to study the Tian-Yau manifold, we must generalize the results of the last section to manifolds defined by the zero-locus of a finite number of homogeneous polynomial equations in a product of projective spaces. Let us first introduce some notation.

The quintic in $\mathbb{C P}^{4}$ is given by

$$
\mathbb{C P}^{4}|5|_{200}
$$

while the Tian-Yau manifold is given by

$$
\begin{array}{l|lll|}
\mathbb{C P}^{3} & 1 & 3 & 0 \\
\mathbb{C P}^{3} & 1 & 0 & 3
\end{array} \mid
$$

In other words, the Tian-Yau manifold is given by three polynomial equations in $\mathbb{C P}^{3} \times \mathbb{C P}^{3}$ one of degree 1 in both $\mathbb{C P}^{3}$, one of degree 3 in the first $\mathbb{C P}^{3}$, and one of degree 3 in the second $\mathbb{C P}^{3}$. That is, if $x_{\mu}$ and $y_{m}$ are respectively homogeneous coordinates of the two $\mathbb{C P}^{3}$, it represents the system of equations

$$
f^{\mu \nu \rho} x_{\mu} x_{\nu} x_{\rho}=0, \quad g^{m n r} y_{m} y_{n} y_{r}=0, \quad h^{\mu n} x_{\mu} y_{m}=0
$$

where $f, g, h$ are coefficients of the equations. 
Therefore, we should say that the Tian-Yau manifold is an element of the configuration above, rather than the configuration itself. In fact, it is given by the following equations with fixed coefficients:

$$
\sum_{i=0}^{3} x_{i} y_{i}=0 \quad \sum_{i=0}^{3}\left(x_{i}\right)^{3}=0 \quad \sum_{i=0}^{3}\left(y_{i}\right)^{3}=0
$$

Now we want to compute the Euler characteristic and the Hodge numbers of this configuration. It is straightforward to generalize the results of the previous section.

Let $\mathrm{X}$ be a smooth complete intersection manifold defined by the configuration matrix

$$
\begin{array}{c|ccc}
\mathbb{C P}^{n_{1}} & d_{1}^{1} & \cdots & d_{N}^{1} \\
\vdots & \vdots & & \vdots \\
\mathbb{C P}^{n_{l}} & d_{1}^{l} & \cdots & d_{N}^{l}
\end{array} \mid
$$

that is it is a complete intersection manifold in a product of 1 projective spaces of dimensions $n_{i}, i=1,2, . ., l$, defined by the zero-locus of $\mathrm{N}$ polynomials of degree vectors $d_{j}, j=1, \ldots, N$ in the 1 projective spaces. Given such a configuration, we can generalize the previous computation of the Chern class to obtain

$$
c(X)=\frac{\prod_{i=1}^{l}\left(1+x_{r}\right)^{n_{r}+1}}{\prod_{a=1}^{N}\left(1+\sum_{s=1}^{l} d_{a}^{s} x_{s}\right)}
$$

By expanding, the first Chern class is

$$
c_{1}(X)=\sum_{i=1}^{l}\left(n_{r}+1-\sum_{a=1}^{N} d_{a}^{r}\right) x_{r}
$$

For $c_{1}(X)$ to be zero, all the coefficients in the sum must vanish, and we find the condition

$$
\sum_{a=1}^{N} d_{a}^{r}=n_{r}+1, \quad \forall r=1, \ldots, l
$$

For the Tian-Yau manifold, $\mathrm{l}=2, n_{1}=n_{2}=3, \mathrm{~N}=3$ and $d_{1}^{1}=d_{1}^{2}=$ $1, d_{2}^{1}=3, d_{2}^{2}=0, d_{3}^{1}=0$ and $d_{2}^{3}=3$. The condition is satisfied, and therefore the Tian-Yau manifold is a Calabi-Yau manifold.

To find its Euler characteristic, we must expand the total Chern class to find an expression for the third Chern class. If $c_{1}(X)=0$ we find that

$$
c_{3}(X)=\sum_{r, s, t=1}^{l}\left(\frac{1}{3}\left[\delta^{r s t}\left(n_{r}+1\right)-\sum_{a=1}^{N} d_{a}^{r} d_{a}^{s} d_{a}^{t}\right]\right) x_{r} x_{s} x_{t}
$$


The Euler characteristic of the Tian-Yau manifold is $\chi=-18$.

Now let us try to find the Hodge numbers of the Tian-Yau manifold. We will use the same method as for the quintic, namely simply counting the free parameters in the polynomial equations.

Two equations are of degree 3 in 4 variables, so together they have 40 free parameters. However, in each $\mathbb{C P}^{3}(16-1)$ of them can be removed by a homogeneous linear change of variables, and 1 by overall rescaling. Therefore in these two equations there are in total 8 free parameters. Now the third equation has 16 coefficients, and 1 can be removed by oversall rescaling. Therefore, in total there are $15+8=23$ free parameters. Hence

Further, from the equation $\chi=2\left(h^{1,1}+h^{2,1}\right)$, we find that the Tian-Yau manifold has Euler characteristic the Euler characteristic is and Hodge diamond

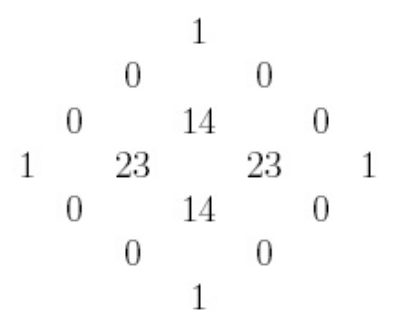

3.4.3. $\mathbb{P}_{5}$. We will construct a Hodge diamond for three-fold Calabi-Yau $X_{3} \in \mathbb{P}_{5}$, which is a complete intersection of a quadric and a quartics:

$$
\begin{array}{r}
X=S^{2} \cap S^{4} \\
h^{i, 1}=(0,1,89,0) \text { and } h^{i, 2}=(0,89,1,0)
\end{array}
$$

The Hodge diamond of complete intersectin of a quadric and a quartic in $\mathbb{P}_{5}$ has the form

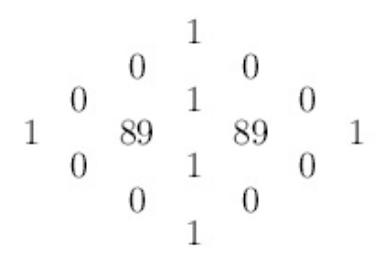

Chern class is

$$
c(X)=\frac{(1+t)^{n+1}}{\prod_{i=1}^{k}\left(1+s_{i} t\right)}
$$

The Euler characteristic is $\chi=-176$

3.5. Calabi-Yau n-folds. Examples of a Calabi-Yau n-folds can be constructed as a submanifold of $\mathbb{C} P^{n+1}$ for all $n>1$. Complex projective space, $\mathbb{C} P^{n}$, sometimes just denoted $P^{n}$, is a compact manifold with $\mathrm{n}$ complex dimensions. It can be constructed by taking $\mathbb{C}^{n+1} / 0$, that is the 
set of $\left(z^{1}, z^{2}, \ldots, z^{n+1}\right)$ where the $z^{i}$ are not all zero and making the identifications

$$
\left(z^{1}, z^{2}, \ldots, z^{n+1}\right) \sim\left(\lambda z^{1}, \lambda z^{2}, \ldots, \lambda z^{n+1}\right)
$$

for any nonzero complex $\lambda \neq 0$. Thus, lines in $\mathbb{C}^{n}+1$ correspond to points in $\mathbb{C} P^{n}$.

$\mathbb{C} P^{n}$ is a Kähler manifold, but it is not a Calabi-Yau manifold. The simplest example is $\mathbb{C} P^{1}$, which is topologically the two-sphere $S^{2}$. Obviously, it does not admit a Ricci-flat metric. The standard metric of $\mathbb{C} P^{n}$, called the Fubini - Study metric, is constructed as follows. First one covers the manifold by $n+1$ open sets given by $z^{a} \neq 0$. Then on each open set one introduces local coordinates.

Examples of CalabiYau manifolds can be obtained as subspaces of complex projective spaces. Specifically, let $\mathrm{G}$ be a homogenous polynomial of degree $\mathrm{k}$ in the coordinates $z^{a}$ of $\mathbb{C}^{n+2}$, that is,

$$
G\left(\lambda z^{1}, \lambda z^{2}, \ldots, \lambda z^{n+2}\right)=\lambda k G\left(z^{1}, \ldots, z^{n+2}\right) .
$$

The submanifold of $\mathbb{C} P^{n+1}$ defined by

$$
G\left(z^{1}, \ldots, z^{n+2}\right)=0
$$

is a compact Kähler manifold with n complex dimensions. This submanifold has vanishing first Chern class for $k=n+2$. One way of obtaining this result is to explicitly compute $c_{1}(X)$. To do so note that $c_{1}(X)$ can be expressed through the volume form since $X$ is Kähler. As a volume form on X one can use the pullback of the (n-1)-power of the Kähler form of $C P^{n+1}$. Another way of obtaining this result is to use the adjunction formula of algebraic geometry, which implies

$$
c_{1}(X) \sim[k-(n+2)] c_{1}\left(\mathbb{C} P^{n+1}\right) .
$$

This vanishes for $\mathrm{k}=\mathrm{n}+2$.

Acknowledgements. We thank the Faculty of Technical Sciences of University Ismail Qemali, Vlora, Albania.

\section{REFERENCES}

[1] Katerin Becker, Melan Becker, John H.Schwarz, StRING THEORY AND M-THEORY, A modern introduction, (2007), 354-370.

[2] Vincent Bouchard, Lectures on complex geometry, CalabiYau manifolds and toric geometry,(2007), 26-40.

[3] V. N. Dumachev, Complete Intersection Calabi-Yau Six-folds, (2015).

[4] Shing-Tung Yau, Calabi-Yau manifold, (2009). 
Department of Mathematics, Faculty of Technical Sciences, University Ismail Qemali - Vlora, Albania.

E-mail address: ndsadikaj@gmail.com

Department of Mathematics, Faculty of Technical Sciences, University Ismail Qemali - Vlora, Albania.

E-mail address: dukaanila@gmail.com 